\title{
Complex Fasciculation Potentials in Amyotrophic Lateral Sclerosis
}

\author{
Toshio Shimizu, MD, PhD ${ }^{1}$ and Kota Bokuda, $\mathrm{MD}^{2}$
}

1. Director and Neurologist; 2. Neurologist, Department of Neurology, Tokyo Metropolitan Neurological Hospital, Tokyo, Japan

\begin{abstract}
Fasciculation and fasciculation potentials (FPS) are most frequently observed in progressive motor neuron disorders, including amyotrophic lateral sclerosis (ALS). Recent diagnostic criteria, the so-called Awaji criteria, emphasize the importance of FPs in the electrodiagnosis of ALS. Although FPS are found in other benign syndromes, FPS in ALS often show a complex morphology (complex fasciculation potential [CFP]) with instability in the form of increased jitters with blocking of some components of the FPS. The criteria have raised the diagnostic significance of FPS as FPs have clinical importance in the diagnosis of ALS equivalent to spontaneous denervation potentials (fibrillation potentials and positive sharp waves). CFPs have multiple pathomechanisms, but they frequently originate from the most distal sites of the peripheral motor neurons. In addition, CFPs are strongly related to axonal membrane hyperexcitability. The increased amount of CFPs in individual patients has been reported to predict shorter survival and rapid progression of the disease. These findings suggest that CFPs have a disease-specific significance to ALS; therefore, exact documentation of CFPs in needle electromyography may contribute to correct diagnoses of ALS and early clinical trials.
\end{abstract}

\section{Keywords}

Amyotrophic lateral sclerosis, complex fasciculation potential, diagnostic criteria, survival prognosis, motor neuron disorders

Disclosure: Toshio Shimizu, MD, PhD, and Kota Bokuda, MD, have no conflicts of interest to declare. Toshio Shimizu, MD, PhD, was supported by a Grant-in-Aid for ALS and Neuropathy Project (2011-2014) from the Tokyo Metropolitan Institute of Medical Science, and by Grants-in-Aid from the Research Committee of CNS Degenerative Diseases (2011-2013) by the Ministry of Health, Labour and Welfare of Japan. No funding was received for the publication of this article.

open Access: This article is published under the Creative Commons Attribution Noncommercial License, which permits any noncommercial use, distribution, adaptation, and reproduction provided the original author(s) and source are given appropriate credit.

Received: February 11, 2015 Accepted: March 7, 2015 Citation: US Neurology, 2015;11(1):53-6 DOI: 10.17925/USN.2015.11.01.53

Correspondence: Toshio Shimizu, MD, PhD, Department of Neurology, Tokyo Metropolitan Neurological Hospital, 2-6-1 Musashidai, Fuchu, Tokyo 183-0042, Japan.

E: toshio_shimizu@tmhp.jp

Fasciculation, which is observed in various peripheral motor neuron disorders, is most frequently found in nonprogressive benign fasciculation syndrome (BFS). ${ }^{1}$ Patients with BFS never exhibit muscle atrophy or pathologic weakness and not all such patients visit hospitals; therefore, the prevalence and characteristics of BFS still need to be established. However, among the progressive disorders involving motor neurons, amyotrophic lateral sclerosis (ALS), progressive muscular atrophy, and other motor neuron diseases such as Kennedy disease, seem to be the most frequent disorders that show overt fasciculation. In ALS, especially, fasciculation is often noted before patients recognize atrophy or weakness. ${ }^{2}$ Acquired neuromyotonia (Isaacs' syndrome) also shows overt fasciculation and myokymia mimicking ALS. ${ }^{3}$ It is often difficult to establish a definite diagnosis of ALS, BFS, or other generalized disorders when patients complain of only fasciculation; in such cases, electrophysiological examinations, including needle electromyography (EMG) and nerve conduction study, are necessary and useful. ${ }^{4}$

\section{Fasciculation Potentials in Amyotrophic Lateral Sclerosis}

Fasciculation in ALS is an important phenomenon for making a definite diagnosis and for considering the pathophysiologic basis of ALS. The fasciculation potential (FP) on needle EMG directly reflects clinical fasciculation, and confirmation of FP is exclusively essential for making a diagnosis. ${ }^{4,5}$ Some patients with ALS do not exhibit FP, even when inspected by a well-trained examiner. However, in patients with typical ALS, FPS usually appear in at least one muscle in a thorough examination with needle EMG. ${ }^{6,7}$

In the revised El Escorial diagnostic criteria of ALS published in $1998,{ }^{8} \mathrm{FPS}$ were described as follows: "Fasciculation potentials are a characteristic clinical feature of ALS. Their presence in EMG recordings is helpful in the diagnosis of ALS, particularly if they are of long duration and polyphasic, and when they are present in muscles in which there is evidence of active or chronic partial denervation and reinnervation. Their distribution can vary. Their absence raises diagnostic doubts, but does not preclude the diagnosis of ALS. Fasciculation potentials of normal morphology occur in normal subjects (benign fasciculations), and fasciculation potentials of abnormal morphology occur in other denervation disorders, e.g. motor neuropathies."

This description is entirely accurate, but clinical experiences have significantly reduced the diagnostic significance of FPS for ALS. While the 

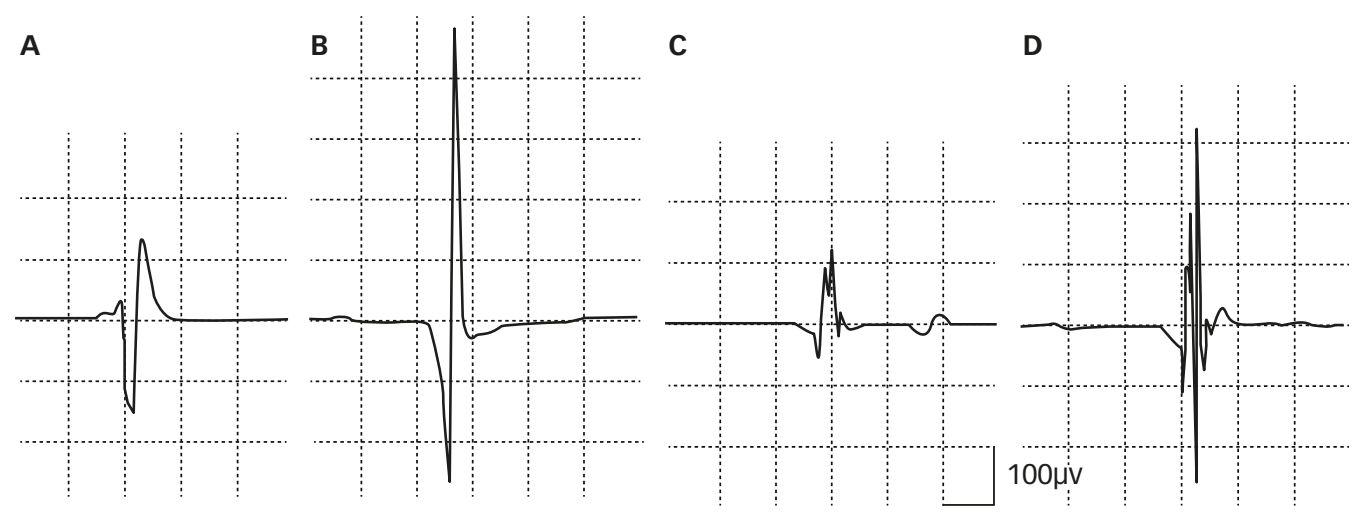

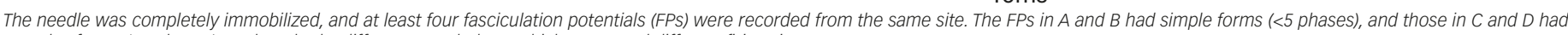
complex forms ( $\geq 5$ phases). Each FP had a different morphology, which suggested different firing sites.

absence of FPS cannot preclude ALS, the presence of FPS does not exclude other disorders. This had led clinicians to reject the significance of FPs in the diagnosis of ALS.

By contrast, the Awaji criteria (2008) emphasize FPs much more than the revised El Escorial criteria. ${ }^{4}$ The Awaji criteria describe FPs as follows: "We propose that the presence of fasciculation potentials (FPS) in a muscle identified as showing needle EMG features of neurogenic change should serve as evidence of ongoing denervation, equivalent in importance to fibrillation potentials and positive sharp waves (fibs-sw). FPs associated with neurogenic disease, especially ALS, show a complex morphology, and often exhibit instability when studied with a high band pass filter and a trigger delay line, which reveals increased jitter with blocking of some components, indicating their origin from reinnervated motor units. 910 When FPs and/or fibs-sw are found in conjunction with chronic neurogenic abnormalities in the context of a diffuse or root-related disorder with superimposed upper motor neuron signs, they assume critical significance and suggest ALS as the diagnosis. We conclude that FPs and fibs-sw have similar and equivalent importance in diagnosis in ALS."

In the process of diagnosing ALS, FPS-especially those with complex morphology-have an equivalent significance to fibrillation potentialspositive sharp waves (fibs-sw) as denervation spontaneous potentials. Furthermore, these criteria define complex FPS (CFPS) as FPs with one feature of polyphasic ( $>4$ phases), increased duration, or increased amplitude compared with the normal values for motor unit potentials (MUPS) in the affected muscle (see Figure 1). CFPs may be unstable. In the clinical setting of an EMG examination, it may be difficult to immediately define increases in the duration and amplitude of FPs; however, the polyphasic feature and instability of FPs may be easy to detect in on-line analyses. Because of the revision in the Awaji criteria, the diagnostic significance of FPS in ALS has been markedly increased, resulting in improvements in the diagnostic rate of ALS. ${ }^{11-14}$

\section{Pathomechanisms of Fasciculation Potential in Amyotrophic Lateral Sclerosis}

FPS have multiple origins, which can be anywhere in the peripheral motor neuron. ${ }^{15-18}$ When FPs originate from a site proximal to axon terminal arborization in the absence of pathologic changes in motor units, FPS should have a simple form, similar to the shape of an MUP (see Figure 2A). ${ }^{15,19,20}$ Simple-form FPs can also originate from the most distal portion of the axon terminal arborization as shown in Figure $2 B$, and a contraction of a few or several muscle fibers results in a simple-form FP. The pathophysiology of FP complexity (CFP) might be explained by the following mechanisms:1,15,18-21 (1) temporal dispersion of MUPS by retrograde transmission of potentials fired distally in axon sprouts with conduction through neighbor branches (see Figure 2C); (2) temporal dispersion of MUPs caused by distal conduction through degenerating branches with slow conduction and blocking (see Figure 2D); (3) double or triple firing at the same or almost same axonal terminal sites (see Figure 2E). In this case, if an interfiring interval is long, it leads to a double discharge. However, if the interfiring interval is short (several milliseconds), it results in CFP. (4) Temporal dispersion of MUPs due to reinnervation of the neighbor motor unit (see Figure 2F); (5) temporal dispersion of MUPS with reinnervation through immature branches with conduction slowing and blocking (see Figure $2 G$ ); and (6) firing of double motor units due to ephatic axonal transmission (see Figure $2 \mathrm{H}$ ). Ephatic transmission was recently certified by de Carvalho et al. who reported that FPs with origins in two different motor units simultaneously appeared in a single needle recording. ${ }^{20}$ As mentioned before, FPS in ALS can originate from any site of the motor neurons; however, FPs firing at most distal sites of peripheral motor neurons are the most frequent.

\section{Fasciculation Potential of Cortical Origin in Amyotrophic Lateral Sclerosis}

It is well-known that ALS shows hyperexcitability of the motor cortex, which is related to the corticomotoneuronal degeneration probably induced by glutamate-related excitotoxicity. ${ }^{22,23}$ The cortical hyperexcitability, shown as intracortical disinhibition by transcranial magnetic stimulation (TMS) studies, might precede lower motor neuron dysfunction in ALS. ${ }^{24}$ There has been some evidence that the motor cortex or upper motor neurons produce fasciculation in muscles. 16,17,22 TMS studies showed stimulation-induced FPS, which were stable with a simple morphology. ${ }^{16}$ Hirota et al. reported the combined FPS in ALS as one form of CFPS, which were not observed in Kennedy disease, suggesting a supraspinal mechanism reflecting dysfunction of the descending motor pathways. ${ }^{17}$ It is unknown whether this combined 
FP is associated with the motor cortical hyperexcitability, but spontaneous and irregular firings of the corticomotor neurons could induce involuntary firings of the lower motor neurons leading to FPS or CFPS in ALS.

\section{Diagnostic Specificity of Complex Fasciculation Potential in Amyotrophic Lateral Sclerosis}

To date, ALS is considered the most representative disorder in which FPS originate from the most distal axons of peripheral motor nerves. However, the specificity of CFPS to ALS has been debatable. Mills reported that there were no significant differences in the morphologies of FPS between ALS and BFS and that CFPS appear as frequently in BFS as ALS. ${ }^{1}$ Even though Mills is one of the authors of the Awaji criteria, he doubted the diagnostic significance of CFPS in ALS. Thereafter, de Carvalho and Swash, who are also the authors of the Awaji criteria, severely opposed Mills' opinion. ${ }^{21}$ They reported the clear differences in FPS between ALS and BFS, and discussed that no complex or unstable FPS were observed in subjects with BFS. In addition, they reported that CFPS in ALS change during stage progression along with changes of MUP morphology due to neuronal degeneration and reinnervation. ${ }^{21}$ The characteristics or disease concept of BFS have not been well established, and there have been reports that patients with BFS progressed to an intermediate form of $\mathrm{ALS}^{2}$ When clinicians encounter patients with only FPS (especially CFPS), thorough EMG and follow-up examinations are necessary for years.

Acquired neuromyotonia (Isaacs' syndrome) also shows FPs and myokymic discharges including doublets (double discharges) and multiplets, which need the differential diagnosis with ALS. ${ }^{3,25}$ Although electromyographic properties of FPS in neuromyotonia have not been fully investigated, the differential diagnosis with ALS is not difficult because of its peculiar clinical characteristics, without muscle wasting and upper motor neuron signs, and the neuromyotonic discharges and few denervation potentials on EMG in acquired neuromyotonia. ${ }^{25,26}$

\section{Prognostic Significance of Complex Fasciculation Potential in Amyotrophic Lateral Sclerosis}

Despite the above discussion, CFPs are most frequently observed in ALS in EMG laboratories. As shown in Figure 2, if CFPs reflect abnormal axonal membrane hyperexcitability of peripheral motor nerves, it might be a phenomenon deeply related to the pathophysiology of ALS. The double firing or retrograde axonal transmission might be related to the augmented excitability of the axonal membrane. Axonal excitability tests have elucidated two kinds of axonal ion channel abnormalities: increased persistent sodium currents and reduced potassium currents, both of which might increase axonal excitability and induce fasciculation. ${ }^{27-29}$ In addition, Kanai et al. reported that an increase in axonal persistent sodium currents in the early stages of the disease was a strong predictor of a shorter survival in ALS. ${ }^{30}$ Furthermore, the increased amount of CFPS in patients has been reported to predict a shorter survival and rapid progression. ${ }^{7,31}$ This observation fits well with the results of Kanai et al. ${ }^{30}$ Although they studied only median nerve excitability, this functional alteration could be present in generalized body regions, and the extent of the excitability abnormalities could be deeply associated with survival prognosis. Presumably, more generalized axonal hyperexcitability of the
Figure 2: Schema for the Pathomechanisms of Fasciculation Potentials

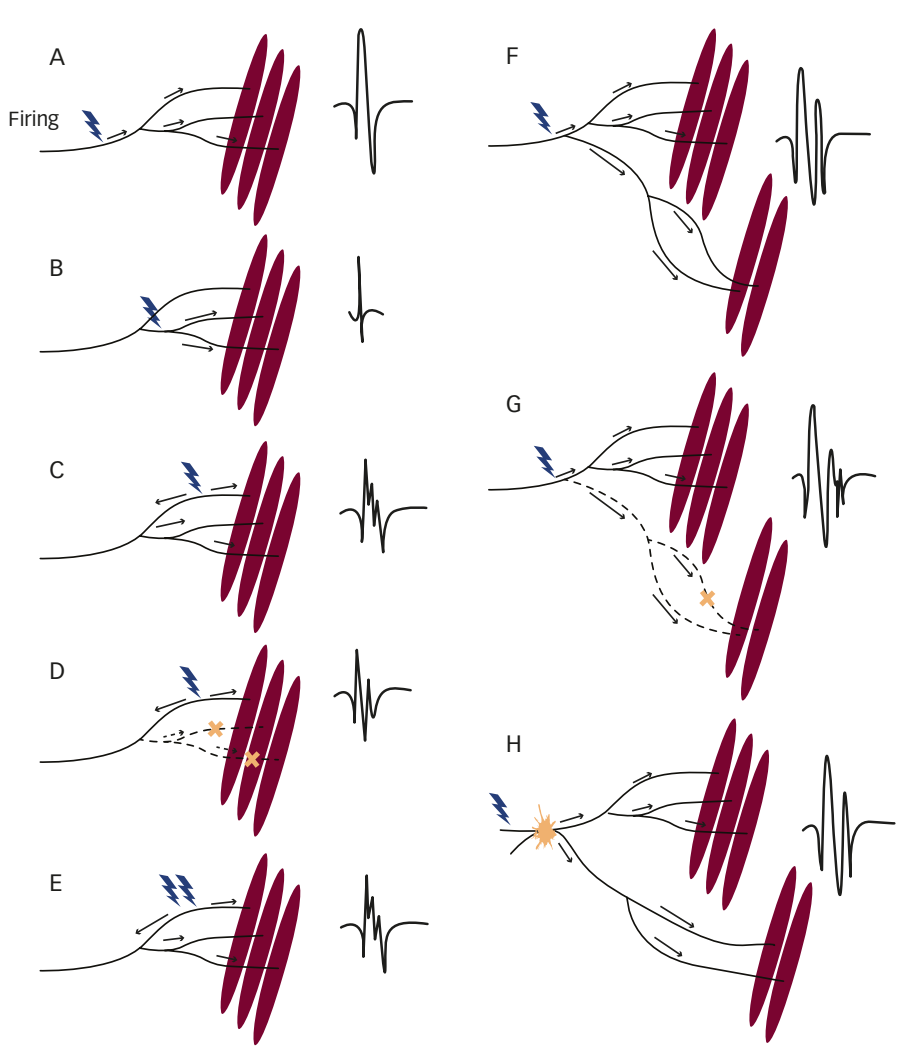

$A$ and $B$ indicate simple-form fasciculation potentials (FPS). $\mathrm{C}$ to $\mathrm{H}$ represent complex form FPS (CFPS). For details, see the text. Reproduced with permission from Bokuda and Shimizu, 2014. ${ }^{19}$

lower motor neurons would produce more frequent FPs, resulting in fast progression and poor survival prognosis.?

In addition, the motor cortical hyperexcitability shown as a reduction in short-interval intracortical inhibition by TMS was reported to correlate with the peripheral axonal hyperexcitability in ALS patients. ${ }^{32}$ Taken together, the hyperexcitability of both the upper and lower motor neurons would produce FPs or CFPs, leading to rapid disease progression.

Contrary to ALS, there have been no reports on the relationship between the amount of FPs or peripheral axonal excitability and disease progression or survival prognosis in acquired neuromyotonia. The spontaneous motor unit activity in neuromyotonia presumably originates from the nerve terminals judging from the normal results of axonal excitability test examined by nerve trunk stimulation. ${ }^{33}$ The immunologic pathophysiology and focal involvements at the nerve terminals in acquired neuromyotonia may be attributable to the differences in disease progression and survival with those of ALS, which has a generalized and neurodegenerative aspect.

\section{Conclusion}

FPS are a classic phenomenon in ALS. However, we have only recently been recognizing their significance as a disease-related biomarker. ${ }^{34}$ In the future, the treatment of ALS may become most important in the earliest stages when patients show only FPs without or with minimal denervation (fibs-sw). Thus, the early and exact documentation of FPS/CFPS in needle EMG and correct diagnoses may have a more crucial significance in the near future. 


\section{Amyotrophic Lateral Sclerosis}

1. Mills KR, Characteristics of fasciculations in amyotrophic lateral sclerosis and the benign fasciculation syndrome, Brain, 2010;133:3458-69

2. de Carvalho M, Swash M, Fasciculation-cramp syndrome preceding anterior horn cell disease: an intermediate syndrome?, J Neurol Neurosurg Psychiatry, 2011;82:459-61.

3. Hart IK, Maddison P, Newsom-Davis J, et al., Phenotypic variants of autoimmune peripheral nerve hyperexcitability, Brain, 2002;125:1887-95.

4. de Carvalho M, Dengler R, Eisen A, et al., Electrodiagnostic criteria for diagnosis of ALS, Clin Neurophysiol, 2008; 119:497-503.

5. Shimizu T, Electrodiagnosis of amyotrophic lateral sclerosis, Ipn J Clin Neurophysiol, 2013;41:94-102

6. Higashihara M, Sonoo M, Imafuku I, et al., Fasciculation potentials in amyotrophic lateral sclerosis and the diagnostic yield of the Awaji algorithm, Muscle Nerve, 2012;45:175-82.

7. Shimizu T, Fujimaki Y, Nakatani-Enomoto S, et al., Complex fasciculation potentials and survival in amyotrophic latera sclerosis, Clin Neurophysiol, 2014:125:1059-64.

8. Brooks BR, Miller RG, Swash M, Munsat TL, El Escorial revisited: revised criteria for the diagnosis of amyotrophic lateral sclerosis, Amyotroph Lateral Scler Other Motor Neuron Disord, 2000;1:293-9.

9. de Carvalho M, Swash M, Fasciculation potentials: a study of amyotrophic lateral sclerosis and other neurogenic disorders, Muscle Nerve, 1998;21:336-44.

10. Janko M, Trontelj JV, Gersak K, Fasciculations in motor neuron disease: discharge rate reflects extent and recency of collateral sprouting, J Neurol Neurosurg Psychiatry, 1989;52:1375-81.

11. Carvalho MD, Swash M, Awaji diagnostic algorithm increases sensitivity of El Escorial criteria for ALS diagnosis, Amyotroph Lateral Scler, 2009;10:53-7.

12. Costa J, Swash M, de Carvalho M, Awaji criteria for the diagnosis of amyotrophic lateral sclerosis:a systematic review,
Arch Neurol, 2012;69:1410-6.

13. Noto Y Misawa S, Kanai K et al., Awaji ALS criteria increase the diagnostic sensitivity in patients with bulbar onset, Clin Neurophysiol, 2012;123:382-5.

14. Okita T, Nodera H, Shibuta Y, et al., Can Awaji ALS criteria provide earlier diagnosis than the revised El Escorial criteria?, J Neurol SCi, 2011;302:29-32.

15. de Carvalho M, Pathophysiological significance of fasciculations in the early diagnosis of ALS, Amyotroph Lateral Scler Other Motor Neuron Disord, 2000;1(Suppl. 1):S43-6.

16. de Carvalho M, Miranda PC, Lourdes Sales Luis M, Ducla-Soares E, Neurophysiological features of fasciculation potentials evoked by transcranial magnetic stimulation in amyotrophic lateral sclerosis, J Neurol, 2000;247:189-94.

17. Hirota N, Eisen A, Weber M, Complex fasciculations and their origin in amyotrophic lateral sclerosis and Kennedy's disease Muscle Nerve, 2000;23:1872-5.

18. Kleine BU, Stegeman DF, Schelhaas HJ, Zwarts MJ, Firing pattern of fasciculations in ALS: evidence for axonal and neuronal origin, Neurology, 2008:70:353-9.

19. Bokuda K, Shimizu T, Fasciculation potentials in ALSsignificance, and relationship with clinical features, Rinsho Shinkeigaku, 2014;54:1083-5.

20. de Carvalho $M$, Swash $M$, Origin of fasciculations in amyotrophic lateral sclerosis and benign fasciculation syndrome, JAMA Neurol, 2013;70:1562-5.

21. de Carvalho M, Swash M, Fasciculation potentials and earliest changes in motor unit physiology in ALS, J Neurol Neurosurg Psychiatry, 2013;84:963-8.

22. Vucic S, Ziemann U, Eisen A, et al., Transcranial magnetic stimulation and amyotrophic lateral sclerosis: pathophysiological insights, I Neurol Neurosurg Psychiatry, 2013;84:1161-70

23. Ziemann $\mathrm{U}$, Winter $\mathrm{M}$, Reimers $\mathrm{CD}$, et al., Impaired motor cortex inhibition in patients with amyotrophic lateral sclerosis.
Evidence from paired transcranial magnetic stimulation, Neurology, 1997:49:1292-8.

24. Menon P, Kiernan MC, Vucic S, Cortical hyperexcitability precedes lower motor neuron dysfunction in ALS, Clin Neurophysiol, 2015;126:803-9.

25. Maddison P, Neuromyotonia, Clin Neurophysiol, 2006;117:2118-27.

26. Maddison P, Mills KR, Newsom-Davis J, Clinica electrophysiological characterization of the acquired neuromyotonia phenotype of autoimmune peripheral nerve hyperexcitability, Muscle Nerve, 2006:33:801-8.

27. Bostock H, Sharief MK, Reid G, Murray NM, Axonal ion channel dysfunction in amyotrophic lateral sclerosis, Brain 1995:118:217-25.

28. Kanai K, Kuwabara S, Misawa S, et al., Altered axonal excitability properties in amyotrophic lateral sclerosis: impaired potassium channel function related to disease stage, Brain, 2006;129:953-62.

29. Vucic S, Kiernan MC, Axonal excitability properties in amyotrophic lateral sclerosis, Clin Neurophysio, 2006:117:1458-66.

30. Kanai K, Shibuya K, Sato Y, et al., Motor axonal excitability properties are strong predictors for survival in amyotrophic lateral sclerosis, I Neurol Neurosurg Psychiatry, 2012;83:734-8.

31. Krarup C, Lower motor neuron involvement examined by quantitative electromyography in amyotrophic lateral sclerosis, Clin Neurophysiol, 2011;122:414-22.

32. Vucic $\mathrm{S}$, Kiernan $\mathrm{MC}$, Upregulation of persistent sodium conductances in familial ALS, I Neurol Neurosurg Psychiatry, 2010;81:222-7.

33. Kiernan MC, Hart IK, Bostock H, Excitability properties of motor axons in patients with spontaneous motor unit activity, J Neurol Neurosurg Psychiatry, 2001;70:56-64

34. Eisen $\mathrm{A}$, Vucic $\mathrm{S}$, Fasciculation potentials: a diagnostic biomarker of early ALS? I Neurol Neurosurg Psychiatry, 2013:84:948. 